\title{
Association of subchondral bone marrow lesion localization with weight-bearing pain in people with knee osteoarthritis: data from the Osteoarthritis Initiative
}

\author{
Koji Aso ${ }^{1,2^{*}}$, Seyed Mohsen Shahtaheri ${ }^{1}$, Daniel F. McWilliams ${ }^{1}$ and David A. Walsh ${ }^{1}$
}

\begin{abstract}
Background: Subchondral bone marrow lesions (BMLs) detected on MRI in knee osteoarthritis (OA) are associated with knee pain. The prevalence and progression of subchondral BMLs are increased by mechanical knee load. However, associations of subchondral BML location with weight-bearing knee pain are currently unknown. In this study, we aim to demonstrate associations of subchondral BML location and size with weight-bearing knee pain in knee OA.

Methods: We analyzed 1412 and 582 varus knees from cross-sectional and longitudinal Osteoarthritis Initiative datasets, respectively. BML scores were semi-quantitatively analyzed with the MRI Osteoarthritis Knee Score for 4 subchondral regions (median and lateral femorotibial, medial and lateral patellofemoral) and subspinous region. Weight-bearing and non-weight-bearing pain scores were derived from WOMAC pain items. Correlation and negative binomial regression models were used for analysis of associations between the BML scores and pain at baseline and changes in the BML scores and changes in pain after 24-month follow-up.

Results: Greater BML scores at medial femorotibial and lateral patellofemoral compartments were associated with greater weight-bearing pain scores, and statistical significance was retained after adjusting for BML scores at the other 4 joint compartments and other OA features, as well as for non-weight-bearing pain, age, sex, and body mass index (BMI) (medial femorotibial; $B=0.08, p=0.02$. patellofemoral; $B=0.13, p=0.01$ ). Subanalysis revealed that greater medial femorotibial BML scores were associated with greater pain on walking and standing $(B=0.11, p=0.01$, and $B=0.10, p=$ 0.04 , respectively). Lateral patellofemoral $B M L$ scores were associated with pain on climbing, respectively $(B=0.14, p=$ 0.02). Increases or decreases over 24 months in BML score in the medial femorotibial compartment were significantly associated with increases or decreases in weight-bearing pain severity after adjusting for non-weight-bearing pain, age, sex, baseline weight-bearing pain, BMl, and BML at the other 4 joint compartments $(B=0.10, p=0.01)$.

Conclusions: Subchondral BML size at the medial femorotibial joint compartment was specifically associated with the severity and the change in weight-bearing pain, independent of non-weight-bearing pain, in knee OA. Specific associations of weight-bearing pain with subchondral BMLs in weight-bearing compartments of the knee indicate that BMLs in subchondral bone contribute to biomechanically induced OA pain.
\end{abstract}

\footnotetext{
* Correspondence: koji.aso@gmail.com

'Pain Centre Versus Arthritis \& NIHR Nottingham Biomedical Research Centre,

University of Nottingham, Nottingham NG5 1PB, UK

${ }^{2}$ Department of Orthopedic Surgery, Kochi Medical School, Kochi University,

185-1 Oko-cho Kohasu, Nankoku 783-8505, Japan
}

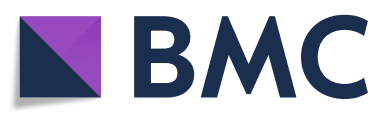

(c) The Author(s). 2021 Open Access This article is licensed under a Creative Commons Attribution 4.0 International License, which permits use, sharing, adaptation, distribution and reproduction in any medium or format, as long as you give appropriate credit to the original author(s) and the source, provide a link to the Creative Commons licence, and indicate if changes were made. The images or other third party material in this article are included in the article's Creative Commons licence, unless indicated otherwise in a credit line to the material. If material is not included in the article's Creative Commons licence and your intended use is not permitted by statutory regulation or exceeds the permitted use, you will need to obtain permission directly from the copyright holder. To view a copy of this licence, visit http://creativecommons.org/licenses/by/4.0/ The Creative Commons Public Domain Dedication waiver (http://creativecommons.org/publicdomain/zero/1.0/) applies to the data made available in this article, unless otherwise stated in a credit line to the data. 


\section{Background}

Knee pain is the major source of disability and reason for hospital visits in patients with knee osteoarthritis (OA). OA knee pain is characteristically experienced during weight-bearing activity, but might also occur at rest on an unloaded joint. Weight-bearing pain indicates biomechanical mechanisms, whereas chemical or neuroplastic mechanisms might dominate in non-weight-bearing pain. Recent accumulating clinical evidence [1-3] indicates that subchondral bone plays a role in generating joint pain in OA. Subchondral bone marrow lesions (BMLs) detected on MRI are increased in people with knee OA compared to non-arthritic controls [1] and are strongly associated with pain. A previous study demonstrated that larger baseline subchondral BMLs were associated with greater baseline knee pain, and increases in total subchondral BML volume were associated with increased knee pain severity [3]. Greater BML scores were associated with weight-bearing pain and less so with non-weightbearing pain [2].

The exact pathophysiology of subchondral BMLs is still under debate. Histologically, subchondral BMLs are characterized by bone marrow necrosis, trabecular abnormalities, bone marrow fibroses, edema, cellular infiltration, and vascular proliferation [4, 5]. Microarray analysis of subchondral BMLs in OA demonstrated upregulation of genes implicated in neurogenesis, osteochondral turnover, and inflammation [5]. Subchondral BMLs might contribute to OA pain by this generation of chemical factors, sensitizing nerves within the subchondral bone. Sensitized subchondral nerves might be activated by biomechanical forces, for example while standing, walking, or climbing stairs. If so, then BMLs in weight-bearing rather than non-weight-bearing regions would be expected to specifically associate with weightbearing pain. However, associations of subchondral BML location with weight-bearing knee pain are currently unknown.

OA structural change particularly affects weightbearing components of the joint and mechanical knee load increases the prevalence and progression of subchondral BMLs [6]. Knee OA is most commonly associated with varus angulation, with increased force and OA structural change affecting the medial femorotibial compartment. Subchondral BMLs present predominantly medially in varus and laterally in valgus lower limbs [7]. Subchondral BMLs typically occur in regions of the joint with the most severe structural change, for example, full-thickness cartilage lesions, and associations between BML scores and cartilage volume were reported [8]. A previous systematic review [6] suggested that a greater medial or lateral load was related to compartmentspecific BMLs. Associations between subchondral BMLs, cartilage damage and varus angulation, and between weight- bearing and non-weight-bearing pain, necessitate careful phenotyping of large numbers of individuals in order to explore specific associations between subchondral BMLs in weight-bearing components of the knee and weight-bearing pain. Longitudinal cohorts can help indicate whether specifically localized subchondral BMLs might mediate weightbearing pain and support the development of BML scores for the stratification of treatments aiming to improve weight-bearing pain.

The purpose of this study was to assess the association of subchondral BML location and size with weightbearing knee pain using the Osteoarthritis Initiative (OAI) dataset. We hypothesized that subchondral BML size at the medial femorotibial joint compartment in people with varus angulation is associated with severity of weight-bearing pain, independent of non-weightbearing pain and the other OA-related MRI features, and that increasing subchondral BML size over time is associated with increased weight-bearing pain severity.

\section{Patients and methods}

\section{Study design and subjects}

Subjects were selected from the OAI, a publicly available multi-center, longitudinal, prospective observational study of knee OA (https://nda.nih.gov/oai). The OAI provides access to the data and images from the OAI longitudinal cohort study and includes data of MRI and radiographic images and Western Ontario and McMaster Universities Osteoarthritis Index (WOMAC) questionnaires [9]. Figure 1 shows a flowchart of participant selection. Of the 4796 participants enrolled in the OAI study, varus knee OA with BML score information of the MRI Osteoarthritis Knee Score (MOAKS) [10], WOMAC questionnaires, femorotibial angle (FTA), and body mass index (BMI) were selected. For subjects with bilateral symptomatic knee OA, one knee was randomly selected using random number generator. We analyzed data from 1412 and 582 knees in the cross-sectional and longitudinal OAI datasets, respectively.

\section{Knee MRI grading}

We used score data from MRI Osteoarthritis Knee Score (MOAKS) at baseline and 24-month follow-up as knee MRI grading. Intra- or inter-rater reliabilities for subchondral BML score data used in this study were very good to excellent (weighted kappa $=0.76-1.00,0.76-$ 0.96, respectively) [10]. For subjects who had multiple score data on the same MRI imaging, the median value was used for this study. In MOAKS, subchondral BMLs were scored in each of the 15 anatomical locations according to percentage of the volume of each BML including volume of any associated cysts as $0=$ none, 1 $<33 \%, 2=33-66 \%$, and $3>66 \%$ using the sagittal 3D double-echo steady-state image [10]. In BML scoring for 


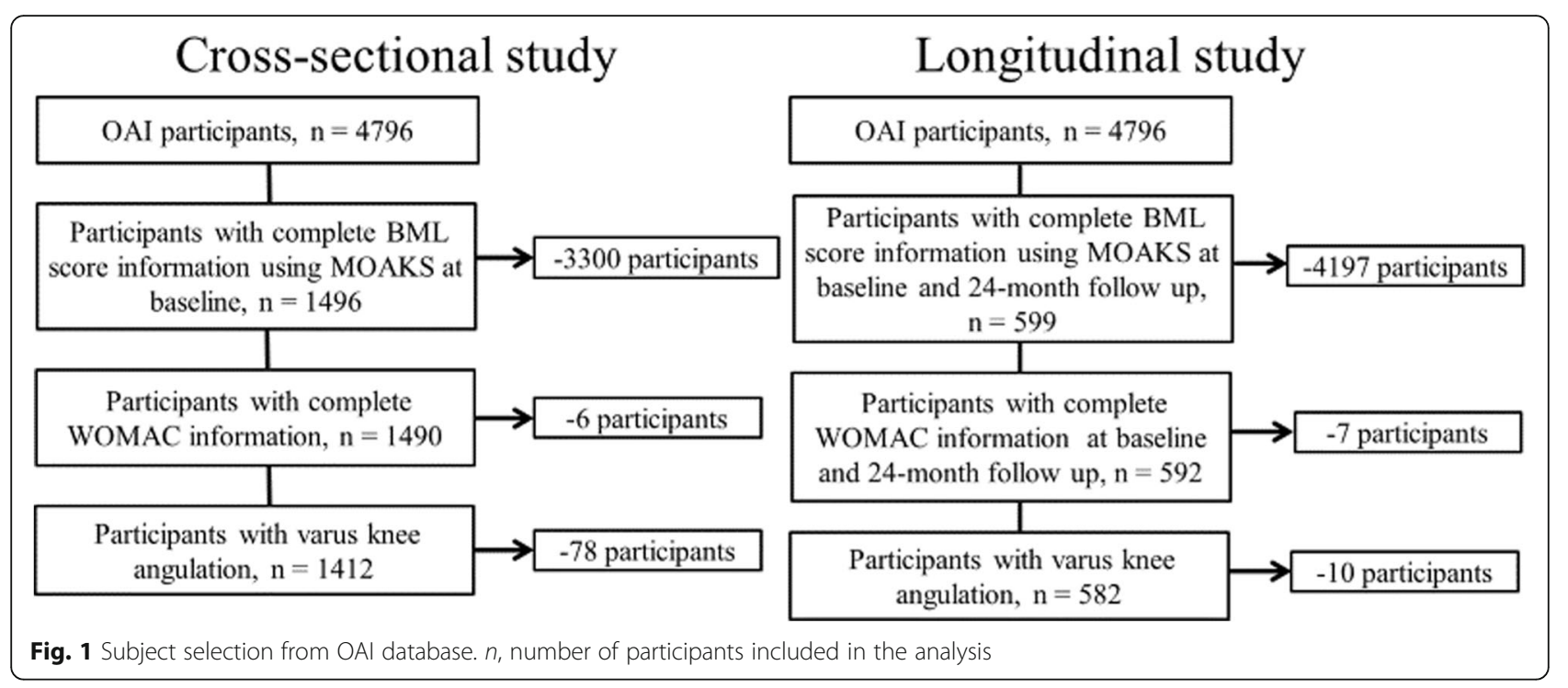

this study, the 15 anatomical locations were integrated into 4 subchondral regions (medial and lateral femorotibial, medial and lateral patellofemoral) and subspinous region (Fig. 2). Articular cartilage, osteophytes, Hoffa's synovitis, effusion, medial meniscus extrusion, and anterior cruciate ligament tears were scored based on MOAKS (supplemental text) [10]. We used the BML scores in the 5 anatomical regions, total cartilage score $(0-42)$, total osteophyte score (0-32), Hoffa's synovitis score (0-3), effusion-synovitis score (0-3), medial meniscus extrusion $(0-3)$, and anterior cruciate ligament tears (absent or present).

\section{Knee pain assessment}

Knee pain was assessed at baseline and 24-month followup using the WOMAC questionnaires. A recent factor analysis of the five WOMAC pain questions suggested that there are two constructs within the five questions [11]. Based on the result, we defined weight-bearing pain as the sum of three WOMAC questions (pain on climbing stairs, on walking, and on standing) and non-weightbearing pain as the sum of two WOMAC questions (pain in bed and when sitting or lying). The time points of interest for cross-sectional and longitudinal study were the baseline visit and baseline and 24-month follow-up visits.
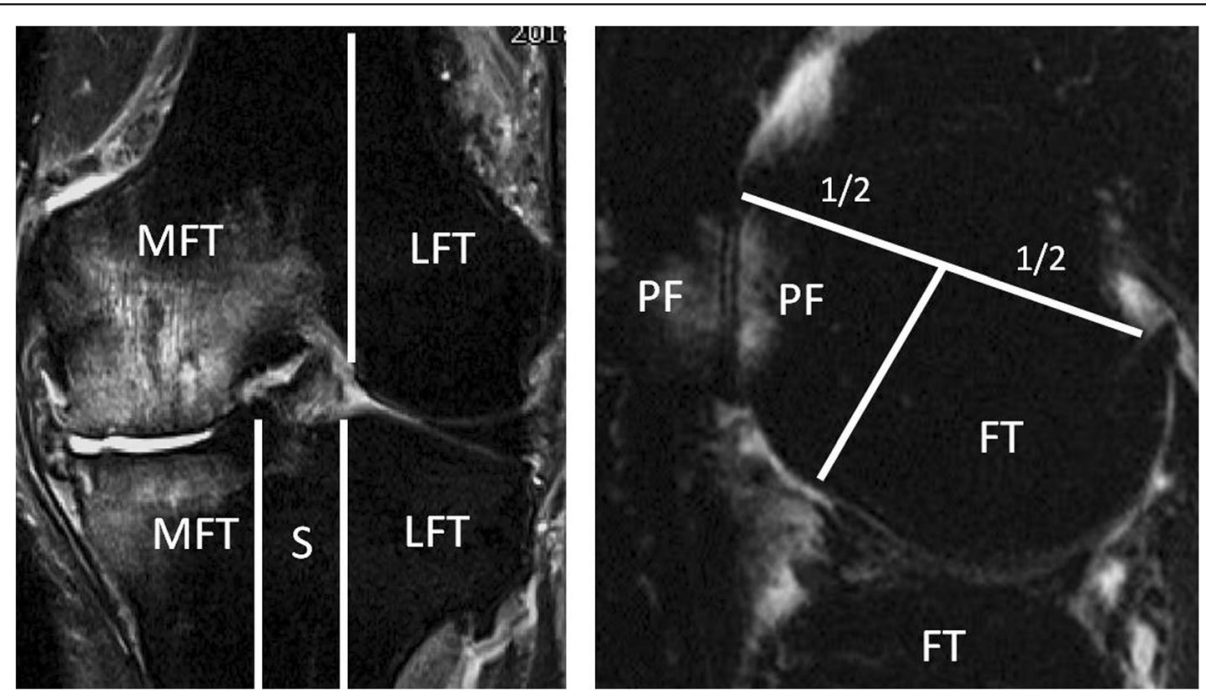

Fig. 2 Delineation of subregional divisions for BML scoring. For BML scoring, the knee was divided into 4 articular sub-regions (medial and lateral femorotibial, and medial and lateral patellofemoral compartment) and subspinous region based on MOAKS; MFT, median femorotibial compartment; LFT, lateral femorotibial compartment; PF, patellofemoral compartment; $S$, subspinous region 


\section{Statistical analysis}

Statistical analyses were performed with SPSS version 26 software. Spearman's correlation coefficients were used for associations between BML score and weightbearing pain. Pearson's correlation coefficients were used for associations between changes in BML scores and changes in weight-bearing pain. Spearman's and Pearson's correlation coefficients and the $p$ values were used for exploratory analysis; therefore, Bonferroni corrections are not used for the correlations. Negative binomial regression was performed for analysis of associations between weight-bearing or non-weightbearing pain or total WOMAC score and scores of BML, articular cartilage, osteophytes, Hoffa's synovitis, effusion, and medial meniscus extrusion. $p<0.05$ indicated statistical significance.

\section{Results}

The participant characteristics in this cross-sectional and longitudinal study are shown in Table 1.

\section{Cross-sectional analyses}

Greater BML, cartilage, osteophyte, Hoffa's synovitis, or effusion-synovitis scores were each associated with greater weight-bearing pain adjusted for non-weight- bearing pain, age, sex, and BMI (supplemental table). Only association between BML scores and weightbearing pain remained significant after adjusting for the other OA-related MRI features and anterior cruciate ligament tears (supplemental table). Separate models explored association of BML localization with weightbearing pain. Greater BML scores at medial femorotibial and lateral patellofemoral compartments were significantly associated with greater weight-bearing pain, and these associations retained significance after adjustment for BML scores at the other 4 joint compartments (Table 2). Subanalysis revealed that greater BML scores at lateral patellofemoral compartments were significantly associated only with greater pain on climbing, whereas the greater scores at medial femorotibial compartments were significantly associated with pain on walking and standing (Table 3).

Greater BML, cartilage, osteophyte, Hoffa's synovitis, or effusion-synovitis scores were each also associated with greater non-weight-bearing pain adjusted for weight-bearing pain, age, sex, and BMI (Table 4). However, only medial meniscus extrusion score remained significantly associated with non-weight-bearing pain after adjusting for the other OA-related MRI features and anterior cruciate ligament tears (Table 4).

Table 1 Participant characteristics at baseline in cross-sectional and longitudinal study

\begin{tabular}{|c|c|c|}
\hline & Cross-sectional study & Longitudinal study \\
\hline Included participants & 1412 & 582 \\
\hline Male (\%) & 38.9 & 42.8 \\
\hline Age (years) & $61.7(8.8)$ & $61.4(8.8)$ \\
\hline BMI $\left(\mathrm{kg} / \mathrm{m}^{2}\right)$ & $29.6(4.9)$ & $30.7(4.8)$ \\
\hline Total BML score, 0-45 & $4.2(3.7)$ & $3.7(2.8)$ \\
\hline BML score at medial FT joint, 0-15 & $1.2(2.0)$ & $1.2(1.7)$ \\
\hline BML score at lateral FT joint, 0-15 & $0.6(1.4)$ & $0.2(0.6)$ \\
\hline BML score at medial PF joint, 0-6 & $0.8(1.0)$ & $0.8(1.0)$ \\
\hline BML score at lateral PF joint, 0-6 & $0.9(1.2)$ & $1.1(1.5)$ \\
\hline BML score at subspinous region, 0-3 & $0.5(0.8)$ & $0.4(0.7)$ \\
\hline Articular cartilage score, 0-42 & $9.0(5.6)$ & $9.0(4.7)$ \\
\hline Osteophyte score, 0-32 & $8.8(6.5)$ & $8.7(6.4)$ \\
\hline Hoffa's synovitis score, 0-3 & $0.7(0.7)$ & $0.7(0.7)$ \\
\hline Effusion-synovitis score, 0-3 & $0.8(0.9)$ & $0.8(0.8)$ \\
\hline Medial meniscus extrusion score, $0-3$ & $0.9(1.0)$ & $1.1(1.0)$ \\
\hline Anterior cruciate ligament tears (\%) & 9.4 & 6.2 \\
\hline FTA (degree) & $185.9(2.6)$ & $186.1(2.0)$ \\
\hline $\mathrm{KL}$ grade $(0 / 1 / 2 / 3 / 4)(\%)$ & 14.0/20.0/32.3/25.3/8.5 & 0/12.7/51.0/36.3/0 \\
\hline Weight-bearing pain, 0-15 & $2.3(2.4)$ & $1.7(2.1)$ \\
\hline Non-weight-bearing pain, 0-10 & $0.9(1.5)$ & $0.7(1.3)$ \\
\hline
\end{tabular}

Data of at baseline in cross-sectional and longitudinal study are displayed as mean (SD) 
Table 2 Cross-sectional associations between OA-related MRI features and weight-bearing pain

\begin{tabular}{|c|c|c|c|c|c|c|}
\hline & Spearman's $r$ & $p$ & $\mathrm{~B}_{1}(95 \% \mathrm{Cl})$ & $p$ & $\mathrm{~B}_{2}(95 \% \mathrm{Cl})$ & $p$ \\
\hline Total BML score & 0.34 & $<0.001^{* *}$ & $0.08(0.04-0.11)$ & $<0.001^{* *}$ & $0.07(0.02-0.11)$ & $0.005^{* *}$ \\
\hline Medial FT joint & 0.29 & $<0.001^{* *}$ & $0.10(0.04-0.16)$ & $0.001^{* *}$ & $0.08(0.02-0.16)$ & $0.02^{*}$ \\
\hline Lateral FT joint & 0.09 & $0.003^{* *}$ & $-0.01(-0.18-0.15)$ & 0.87 & $-0.03(-0.19-0.14)$ & 0.74 \\
\hline Medial PF joint & 0.03 & 0.35 & $0.06(-0.06-0.17)$ & 0.32 & $0.06(-0.06-0.18)$ & 0.31 \\
\hline Lateral PF joint & 0.13 & $<0.001^{* *}$ & $0.14(0.05-0.23)$ & $0.003^{* *}$ & $0.13(0.03-0.23)$ & $0.01^{*}$ \\
\hline Subspinous region & 0.18 & $<0.001^{* *}$ & $-0.04(-0.21-0.14)$ & 0.69 & $-0.06(-0.24-0.12)$ & 0.53 \\
\hline Articular cartilage score & 0.32 & $<0.001^{* *}$ & $0.05(0.01-0.1)$ & $0.03^{*}$ & $0.02(-0.01-0.05)$ & 0.25 \\
\hline Osteophytes score & 0.26 & $<0.001^{* *}$ & $0.04(0.01-0.07)$ & $0.01^{*}$ & $0.01(-0.01-0.03)$ & 0.46 \\
\hline Hoffa's synovitis score & 0.18 & $<0.001^{* *}$ & $0.3(0.03-0.60)$ & $0.03^{*}$ & $-0.03(-0.21-0.15)$ & 0.72 \\
\hline Effusion score & 0.24 & $<0.001^{* *}$ & $0.4(0.13-0.59)$ & $0.002^{* *}$ & $0.08(-0.06-0.22)$ & 0.26 \\
\hline Medial meniscus extrusion score & 0.22 & $<0.001^{* *}$ & $0.15(-0.05-0.36)$ & 0.14 & $0.03(-0.12-0.13)$ & 0.96 \\
\hline
\end{tabular}

$\mathrm{B}_{1}$ : OA-related MRI features adjusted for non-weight-bearing pain, age, sex, and BMI. BML subscores were in addition adjusted for BML at the other four joint compartments. $\mathrm{B}_{2}$ : OA-related MRI features adjusted for non-weight-bearing pain, age, sex, BMI, the other OA-related MRI features, and anterior cruciate ligament tears. BML subscores were in addition adjusted for BML at the other four joint compartments. ${ }^{*} p<0.05,{ }^{* *} p<0.01$

$B M L$ bone marrow lesion, FT femorotibial, PF patellofemoral

\section{Longitudinal analyses}

Changes from baseline to 24-month follow-up of variables in the longitudinal study are shown in Table 5. The increase or decrease in total and site-specific BML scores were associated with increase or decrease in weight-bearing pain severity, respectively. Specifically, the change in BML score at medial femorotibial compartments was significantly associated with change in weight-bearing pain after adjusting for sex, baseline weight-bearing pain, age, BMI, BML at the other four joint compartments, and change in non-weight-bearing pain (Table 6).

No significant longitudinal association between change in medial meniscus extrusion score and change in nonweight-bearing pain was found after adjusting for sex, baseline non-weight-bearing pain, age, BMI and medial meniscus extrusion score, and change in weight-bearing pain $(B=0.15(95 \% \mathrm{CI},-0.15-0.44) p=0.33)$.

\section{Discussion}

In cross-sectional analyses, we firstly demonstrated that subchondral BML size at the medial femorotibial joint compartment in varus knee OA participants was associated with weight-bearing pain severity and that this association was not explained by non-weightbearing pain, other OA-related MRI features, age, sex, or BMI. In longitudinal analyses, increasing or decreasing subchondral BML size at the medial femorotibial joint compartment was associated with increased or decreased weight-bearing pain severity. Our findings support the hypothesis that subchondral BMLs increase OA knee pain due to biomechanical factors acting through the affected subchondral bone.

Greater BML scores at medial femorotibial compartments were significantly associated with greater weightbearing pain, especially pain on walking and standing, even after adjusting for non-weight-bearing pain and the other OA-related MRI features. The increase or decrease in BML score at the medial femorotibial compartment was significantly associated with increased or decreased weight-bearing pain severity. In contrast to these findings, BML scores were not significantly associated with non-weight-bearing pain in our fully adjusted models. Hence, we conclude that subchondral BMLs at weight-

Table 3 Cross-sectional associations between site-specific BML score and pain on climbing stairs, on walking, and on standing

\begin{tabular}{|c|c|c|c|c|c|c|}
\hline \multirow{2}{*}{$\begin{array}{l}\text { Site-specific BML } \\
\text { scores }\end{array}$} & \multicolumn{2}{|l|}{ Pain on climbing } & \multicolumn{2}{|l|}{ Pain on walking } & \multicolumn{2}{|l|}{ Pain on standing } \\
\hline & B (95\% Cl) & $p$ & B (95\% Cl) & $p$ & B (95\% Cl) & $p$ \\
\hline Medial FT joint & $0.06(-0.02-0.14)$ & 0.13 & $0.11(0.02-0.20)$ & $0.01^{*}$ & $0.10(0.04-0.20)$ & $0.04^{*}$ \\
\hline Lateral FT joint & $0.01(-0.18-0.20)$ & 0.91 & $-0.15(-0.40-0.11)$ & 0.26 & $-0.01(-0.26-0.23)$ & 0.93 \\
\hline Medial PF joint & $0.13(-0.004-0.26)$ & 0.06 & $-0.07(-0.24-0.11)$ & 0.45 & $-0.05(-0.22-0.13)$ & 0.62 \\
\hline Lateral PF joint & $0.14(0.02-0.25)$ & $0.02^{*}$ & $0.08(-0.06-0.22)$ & 0.28 & $0.12(-0.03-0.26)$ & 0.10 \\
\hline Subspinous region & $0.03(-0.18-0.23)$ & 0.80 & $-0.10(-0.35-0.16)$ & 0.46 & $-0.20(-0.48-0.07)$ & 0.13 \\
\hline
\end{tabular}

B: BML subscores adjusted for BML at the other four joint compartments, non-weight-bearing pain, age, sex, BMI, the other OA-related MRI features and anterior cruciate ligament tears. ${ }^{*} p<0.05$

$B M L$ bone marrow lesion, $F T$ femorotibial, $P F$ patellofemoral 
Table 4 Cross-sectional associations between OA-related MRI features and non-weight-bearing pain

\begin{tabular}{|c|c|c|c|c|c|c|}
\hline & Spearmans' $r$ & $p$ & $\mathrm{~B}_{1}(95 \% \mathrm{Cl})$ & $p$ & $\mathrm{~B}_{2}(95 \% \mathrm{Cl})$ & $p$ \\
\hline Total BML score & 0.19 & $<0.001^{* *}$ & $-0.01(-0.06-0.04)$ & 0.63 & $-0.04(-0.10-0.02)$ & 0.21 \\
\hline Articular cartilage score & 0.22 & $<0.001^{* *}$ & $0.01(-0.02-0.04)$ & 0.45 & $-0.01(-0.06-0.04)$ & 0.65 \\
\hline Osteophytes score & 0.20 & $<0.001^{* *}$ & $0.02(-0.01-0.05)$ & 0.15 & $0.02(-0.01-0.05)$ & 0.15 \\
\hline Hoffa's synovitis score & 0.11 & $<0.001^{* *}$ & $0.08(-0.16-0.32)$ & 0.52 & $0.06(-0.20-0.31)$ & 0.68 \\
\hline Effusion score & 0.18 & $<0.001^{* *}$ & $-0.02(-0.18-0.18)$ & 0.99 & $-0.01(-0.004-0.36)$ & 0.90 \\
\hline Medial meniscus extrusion score & 0.20 & $<0.001^{*}$ & $0.16(0.01-0.32)$ & $0.04^{*}$ & $0.18(-0.55-0.68)$ & $0.045^{*}$ \\
\hline
\end{tabular}

$\mathrm{B}_{1}$ : OA-related MRI features adjusted for weight-bearing pain, age, sex and BMI. $\mathrm{B}_{2}$ : OA-related MRI features adjusted for weight-bearing pain, age, sex, BMI, the other OA-related MRI features, and anterior cruciate ligament tears. ${ }^{*} p<0.05,{ }^{* *} p<0.01$

$B M L$ bone marrow lesion, FT femorotibial, PF patellofemoral

bearing components are specifically associated with weight-bearing pain. We previously demonstrated that subchondral bone histopathology characteristic of subchondral BMLs, occurring in middle third of medial tibial plateau (an important weight-bearing area), was associated with knee OA pain, not dependent on chondropathy and synovitis [12].

In this study, subchondral BMLs at the lateral patellofemoral joint compartment were also associated with weight-bearing pain, especially pain on climbing. The associations of patellar BMLs with any knee pain and with pain when going up or down stairs have been reported [13]. The patellofemoral joint is one of the most commonly affected compartments in knee OA, and varus knee deformity has been associated with worsening of patellofemoral OA, especially in the lateral facet [14].

Table 5 Changes in MRI scores, femorotibial angle, and pain between baseline and 24-month follow-up $(n=582)$

\begin{tabular}{|c|c|}
\hline Characteristic (units or possible range) & Mean change (range) \\
\hline BMI $\left(\mathrm{kg} / \mathrm{m}^{2}\right)$ & $0.02(-10.5$ to 13.1$)$ \\
\hline Total BML score, 0-45 & $0.7(-7$ to 12$)$ \\
\hline BML score at medial FT joint, 0-15 & $0.5(-4$ to 10$)$ \\
\hline BML score at lateral FT joint, 0-15 & $0.1(-2$ to 5$)$ \\
\hline BML score at medial PF joint, 0-6 & $0.03(-5$ to 3$)$ \\
\hline BML score at lateral PF joint, 0-6 & $0.1(-3$ to 3$)$ \\
\hline BML score at subspinous region, $0-3$ & 0.07 ( -3 to 3$)$ \\
\hline Articular cartilage score, $\mathbf{0 - 4 2}$ & $0.8(-10$ to 9$)$ \\
\hline Osteophyte score, 0-32 & $0.4(-12$ to 14$)$ \\
\hline Hoffa's synovitis score, 0-3 & $0.08(-2$ to 2$)$ \\
\hline Effusion-synovitis score, 0-3 & $0.1(-2$ to 2$)$ \\
\hline Medial meniscus extrusion score, 0-3 & $0.2(-2$ to 3$)$ \\
\hline New anterior cruciate ligament tears (\%) & 0.7 \\
\hline FTA (degree) & $0.4(-3.9$ to 2.0$)$ \\
\hline Weight-bearing pain, 0-15 & 0.5 (-10 to 10$)$ \\
\hline Non-weight-bearing pain, 0-10 & $0.02(-11$ to 13$)$ \\
\hline
\end{tabular}

Positive values represent increases from baseline to follow- up $B M I$ body mass index, BML bone marrow lesion, FTA femorotibial angle, $F T$ femorotibial, $P F$ patellofemoral
We extend these findings to identify that subchondral BMLs at the lateral and not at medial patellofemoral joint compartments were significantly associated with the severity of weight-bearing pain in people with varus angulation. This again suggests a biomechanical explanation linking subchondral BMLs to OA pain. However, patellofemoral BML score change was not associated with pain change, suggesting that subchondral BML changes at the patellofemoral joint has a smaller impact on changes in weight-bearing pain than does subchondral BML change at the femorotibial joint.

Subchondral BMLs represent regions of subchondral bone characterized as displaying increased bone turnover, and expression of factors, including pro-inflammatory cytokines, that can increase nerve sensitization [5]. We previously showed that nerve growth factor expression within osteochondral channels, and subchondral osteoclast density, each is associated with knee OA pain [12], and calcitonin gene-related peptide immunoreactive sensory nerves within osteochondral channels are associated with pain in human and rat knee OA [15]. Activation of sensory nerves in subchondral bone might contribute to weight-bearing pain in knee OA. Subchondral BMLs might be an imaging biomarker for pathology which sensitizes subchondral nerves and therefore increases weightbearing pain in knee OA. Further investigation is needed to clarify the cellular and molecular factors which might mediate the observed association between subchondral BMLs and weight-bearing pain.

Our results suggested that subchondral BMLs might mediate mechanically induced pain such as during weight-bearing. Biomechanical factors may reciprocally contribute to the pathogenesis of subchondral BMLs. Previous studies [6] reported that increased mechanical load due to malalignment of the knee joint is a risk factor for incident or enlarging subchondral BMLs in the femorotibial joint. Greater BMI that increases the mechanical load on the knee was also associated with increased BMLs size [16]. History of knee injury (e.g., ACL rupture) has been associated with tibiofemoral BMLs [17]. Biomechanical alterations were persistent 
Table 6 Associations between changes in BML scores and changes in weight-bearing pain

\begin{tabular}{lllll}
\hline BML score changes & Pearson's $\boldsymbol{r}$ & $\boldsymbol{p}$ & $\mathbf{B}(\mathbf{9 5 \%} \mathbf{C l})$ & $\mathbf{p}$ \\
\hline Total score & 0.13 & $0.002^{*}$ & $0.07(0.00-0.13)$ & $0.04^{*}$ \\
Medial FT joint & 0.16 & $<0.001^{* *}$ & $0.10(0.02-0.18)$ & $0.01^{*}$ \\
Lateral FT joint & -0.06 & 0.13 & $-0.21(-0.53-0.11)$ & 0.20 \\
Medial PF joint & -0.01 & 0.73 & $-0.10(-0.31-0.11)$ & 0.34 \\
Lateral PF joint & 0.02 & 0.58 & $0.10(-0.09-0.30)$ & 0.29 \\
Subspinous region & 0.10 & $0.01^{*}$ & $0.21(-0.03-0.45)$ & 0.09
\end{tabular}

B: Model for total BML score adjusted for sex, baseline weight-bearing pain, age, and BMI, total BML score, and change in non-weight-bearing pain. BML subscores in addition adjusted for BML at the other four joint compartments. ${ }^{*} p<0.05,{ }^{* *} p<0.01$

$B M L$ bone marrow lesion, $F T$ femorotibial, $P F$ patellofemoral

even after ACL reconstruction [18], and therefore, the altered biomechanical forces might be involved in incident or enlarging subchondral BMLs. Meniscus and cartilage can be shock absorbers that protect subchondral bone from overloading. Associations between meniscal pathology $[19,20]$ or cartilage loss $[19,21]$ and increasing subchondral BML size might be mediated by altered biomechanical forces through the subchondral bone. Some have suggested that strenuous physical activity might increase subchondral BML size by repetitive mechanical load on the knee. Strenuous physical activity was associated with increased subchondral BML size [19], but, on the other hand, non-strenuous activity was negatively associated with subchondral BML size [19, 22]. Different levels of physical activity may have different influences on subchondral bone.

Biomechanical unloading by patellofemoral bracing reduced subchondral BML volume at patellofemoral joint compartments in patients with painful patellofemoral OA [23]. However, lateral wedge insoles, which might reduce the load in the medial femorotibial joint compartment during walking [24], did not significantly changes in subchondral BMLs and pain in medial knee OA [25], and the extent of biomechanical unloading that is required to reverse subchondral BML pathology remains uncertain. Treatments with large effects on mechanical load such as high tibial osteotomy and valgus bracing might be needed to reduce subchondral BMLs at medial femorotibial joint compartments.

Pharmacotherapy also has potential to reduce subchondral BMLs. The bisphosphonate zoledronic acid reduced subchondral BML size and knee pain in people with OA [26]. However, recent meta-analysis [27] and randomized controlled trial [28] did not support analgesic effects of bisphosphonates in knee OA. These studies support that biomechanical unloading is more effective than current pharmacotherapy for subchondral BML associated pain. Knee OA pain has multiple sources, and targeting subchondral BMLs would be expected to have greatest benefit in those cases where subchondral BMLs are the predominant driver of weight-bearing pain.
Subchondral BMLs are also found in healthy pain-free young-middle aged adults, with a prevalence of $13-17 \%$ $[19,29,30]$, which is less than that in symptomatic knee OA. Previous study using animal OA models reported that the first sign of change in the development of OA was subchondral BML which preceded cartilage degeneration [31]. These findings suggest that subchondral BMLs may be involved in the pathogenesis of knee OA before it becomes clinically apparent.

In this study, medial meniscus extrusion score was significantly associated with non-weight-bearing pain severity after adjusting for weight-bearing pain and the other OA-related MRI changes. A recent study reported that patients with medial submeniscal flap tear complained of pains during sleep, but not during daytime activities [32]. Pain associated with meniscal tears might be caused by increased mechanical load on meniscus not only during knee loading but also during unloaded knee flexion-extension motion. However, change in meniscus extrusion score was not associated with change in nonweight-bearing pain, which suggests that meniscus extrusion may have a relative small impact on non-weight-bearing pain.

Not only subchondral BMLs, but also synovitis [33], effusion-synovitis [34], cartilage defects [35], and osteophytes [36] have previously been associated with knee OA pain. We also demonstrated that subchondral BML, osteophyte, and effusion scores were independently associated with total pain score, even after adjusting for the other OA-related MRI features (supplemental table). Associations of pain with cartilage and Hoffa's synovitis scores did not retain statistical significance after adjusting for the other OA-related MRI features including BML score, suggesting that some of these observed associations may be explained by other closely associated OA features (supplemental table).

Our study has several potential limitations. We investigated only 2 time points (baseline and 24 months). A previous study using OAI dataset demonstrated that changes in total subchondral BML volume after 24 months were positively associated with changes in knee 
pain severity [3]. However, multiple assessments with shorter intervals are needed to evaluate how fluctuations of subchondral BML size relate to changes in weightbearing pain. Subchondral BML size was assessed semiquantitatively and, although our study had a large sample size, quantitative BML measurements might have provided additional information. Direct measurement of mechanical loading through joint compartments was not possible in the current study, and interventional studies would be required to confirm our conclusions that subchondral BMLs are biomarkers for pathology which causes biomechanically induced nociceptive pain.

\section{Conclusions}

We demonstrated that subchondral BML size at the medial femorotibial joint compartment in varus knee OA was associated with weight-bearing pain and that this association was specific for weight-bearing rather than non-weight-bearing pain and was over and above any effects of other OA-related MRI features, age, sex, and BMI. Our findings suggest that specific association of weight-bearing pain with subchondral BMLs in weight-bearing compartments of the knee indicates that subchondral BMLs aggravate biomechanical factors leading to OA pain.

\section{Supplementary Information}

The online version contains supplementary material available at https://doi. org/10.1186/s13075-021-02422-0.

Additional file 1.

Additional file 2.

\section{Acknowledgements}

We would like to thank the OAl participants and Coordinating Center for their work in generating the clinical and radiological data of the OAl cohort and for making them publicly available.

\section{Authors' contributions}

All authors approved the final version to be published. K.A. has full access to all of the data in the study and takes responsibility for the integrity of the data and the accuracy of the data analysis. K.A., M.S., D.M., and D.W. designed the study and analyzed and interpreted the results. K.A., D.M., and D.W. wrote the manuscript.

\section{Funding}

This study was supported by a grant from Versus Arthritis (Centre initiative grant number 20777).

\section{Availability of data and materials}

The datasets analyzed during this study are available from the Osteoarthritis Initiative website (https://nda.nih.gov/oai/).

\section{Ethics approval and consent to participate}

The OAl study was approved by the Institutional Review Board of the University of California, San Francisco, and its affiliates. All participants gave written informed consent.

Consent for publication

All authors agreed to the publication of this manuscript.

\section{Competing interests}

D.A. Walsh: Grants from Versus Arthritis, while the study was being conducted; grants from Pfizer Ltd., other from Pfizer Ltd., personal fees from GlaxoSmithKline, outside the submitted work.

D. F. McWilliams: grants from Pfizer Ltd.

The remaining authors have no conflicts of interest to declare.

Received: 10 July 2020 Accepted: 6 January 2021

Published online: 19 January 2021

\section{References}

1. Stahl R, Jain SK, Lutz J, Wyman BT, Le Graverand-Gastineau MP, Vignon E, Majumdar S, Link TM. Osteoarthritis of the knee at 3.0 T: comparison of a quantitative and a semi-quantitative score for the assessment of the extent of cartilage lesion and bone marrow edema pattern in a 24-month longitudinal study. Skelet Radiol. 2011;40(10):1315-27.

2. Lo GH, McAlindon TE, Niu J, Zhang Y, Beals C, Dabrowski C, Le Graverand $\mathrm{MP}$, Hunter DJ. Bone marrow lesions and joint effusion are strongly and independently associated with weight-bearing pain in knee osteoarthritis: data from the osteoarthritis initiative. Osteoarthr Cartil. 2009;17(12):1562-9.

3. Driban JB, Price L, Lo GH, Pang J, Hunter DJ, Miller E, Ward RJ, Eaton CB, Lynch JA, McAlindon TE. Evaluation of bone marrow lesion volume as a knee osteoarthritis biomarker--longitudinal relationships with pain and structural changes: data from the osteoarthritis initiative. Arthritis Res Ther. 2013;15(5):R112.

4. Hunter DJ, Gerstenfeld L, Bishop G, Davis AD, Mason ZD, Einhorn TA, Maciewicz RA, Newham P, Foster M, Jackson S, et al. Bone marrow lesions from osteoarthritis knees are characterized by sclerotic bone that is less well mineralized. Arthritis Res Ther. 2009;11(1):R11.

5. Kuttapitiya A, Assi L, Laing K, Hing C, Mitchell P, Whitley G, Harrison A, Howe FA, Ejindu V, Heron C, et al. Microarray analysis of bone marrow lesions in osteoarthritis demonstrates upregulation of genes implicated in osteochondral turnover, neurogenesis and inflammation. Ann Rheum Dis. 2017;76(10):1764-73

6. Beckwee D, Vaes $P$, Shahabpour M, Muyldermans R, Rommers $N$, Bautmans I. The influence of joint loading on bone marrow lesions in the knee: a systematic review with meta-analysis. Am J Sports Med. 2015;43(12):3093-107.

7. Felson DT, McLaughlin S, Goggins J, LaValley MP, Gale ME, Totterman S, Li W, Hill C, Gale D. Bone marrow edema and its relation to progression of knee osteoarthritis. Ann Intern Med. 2003;139(5 Pt 1):330-6.

8. Wildi LM, Raynauld JP, Martel-Pelletier J, Abram F, Dorais M, Pelletier JP. Relationship between bone marrow lesions, cartilage loss and pain in knee osteoarthritis: results from a randomised controlled clinical trial using MRI. Ann Rheum Dis. 2010;69(12):2118-24.

9. Bellamy N, Buchanan WW, Goldsmith CH, Campbell J, Stitt LW. Validation study of WOMAC: a health status instrument for measuring clinically important patient relevant outcomes to antirheumatic drug therapy in patients with osteoarthritis of the hip or knee. J Rheumatol. 1988;15(12): 1833-40.

10. Hunter DJ, Guermazi A, Lo GH, Grainger AJ, Conaghan PG, Boudreau RM, Roemer FW. Evolution of semi-quantitative whole joint assessment of knee OA: MOAKS (MRI osteoarthritis knee score). Osteoarthr Cartil. 2011;19(8):990-1002.

11. Stratford PW, Kennedy DM, Woodhouse L, Spadoni GF. Measurement properties of the WOMAC LK 3.1 pain scale. Osteoarthr Cartil. 2007;15(3): 266-72.

12. Aso K, Shahtaheri SM, Hill R, Wilson D, McWilliams DF, Walsh DA. Associations of symptomatic knee osteoarthritis with Histopathologic features in Subchondral bone. Arthritis Rheumatol. 2019;71(6):916-24.

13. Wang J, Antony B, Zhu Z, Han W, Pan F, Wang X, Jin X, Liu Z, Cicuttini F, Jones $\mathrm{G}$, et al. Association of patellar bone marrow lesions with knee pain, patellar cartilage defect and patellar cartilage volume loss in older adults: a cohort study. Osteoarthr Cartil. 2015;23(8):1330-6.

14. Otsuki S, Nakajima M, Okamoto Y, Oda S, Hoshiyama Y, lida G, Neo M. Correlation between varus knee malalignment and patellofemoral osteoarthritis. Knee Surg Sports Traumatol Arthrosc. 2016;24(1):176-81.

15. Aso K, Shahtaheri SM, Hill R, Wilson D, McWilliams DF, Nwosu LN, Chapman $\mathrm{V}$, Walsh DA. Contribution of nerves within osteochondral channels to osteoarthritis knee pain in humans and rats. Osteoarthr Cartil. 2020;28(9): $1245-54$. 
16. Lim YZ, Wang Y, Wluka AE, Davies-Tuck ML, Hanna F, Urquhart DM, Cicuttini FM. Association of obesity and systemic factors with bone marrow lesions at the knee: a systematic review. Semin Arthritis Rheum. 2014;43(5):600-12.

17. Khan HI, Aitken D, Blizzard L, Ding C, Pelletier JP, Pelletier JM, Cicuttini F, Jones G. History of knee injury and MRI-assessed knee structures in middleand older-aged adults: a cross-sectional study. Clin Rheumatol. 2015;34(8): $1463-72$.

18. Amano K, Pedoia V, Su F, Souza RB, Li X, Ma CB. Persistent biomechanical alterations after $\mathrm{ACL}$ reconstruction are associated with early cartilage matrix changes detected by quantitative MR. Orthop J Sports Med. 2016; 4(4):2325967116644421.

19. Antony B, Venn A, Cicuttini F, March L, Blizzard L, Dwyer T, Halliday A, Cross $M$, Jones $G$, Ding $C$. Correlates of knee bone marrow lesions in younger adults. Arthritis Res Ther. 2016;18:31

20. Englund M, Guermazi A, Roemer FW, Yang M, Zhang Y, Nevitt MC, Lynch JA, Lewis CE, Torner J, Felson DT. Meniscal pathology on MRI increases the risk for both incident and enlarging subchondral bone marrow lesions of the knee: the MOST study. Ann Rheum Dis. 2010;69(10):1796-802.

21. Hunter DJ, Zhang Y, Niu J, Goggins J, Amin S, LaValley MP, Guermazi A, Genant H, Gale D, Felson DT. Increase in bone marrow lesions associated with cartilage loss: a longitudinal magnetic resonance imaging study of knee osteoarthritis. Arthritis Rheum. 2006;54(5):1529-35.

22. Racunica TL, Teichtahl AJ, Wang Y, Wluka AE, English DR, Giles GG, O'Sullivan R, Cicuttini FM. Effect of physical activity on articular knee joint structures in community-based adults. Arthritis Rheum. 2007;57(7):1261-8.

23. Callaghan MJ, Parkes MJ, Hutchinson CE, Gait AD, Forsythe LM, Marjanovic EJ, Lunt M, Felson DT. A randomised trial of a brace for patellofemoral osteoarthritis targeting knee pain and bone marrow lesions. Ann Rheum Dis. 2015;74(6):1164-70.

24. Hinman RS, Payne C, Metcalf BR, Wrigley TV, Bennell KL. Lateral wedges in knee osteoarthritis: what are their immediate clinical and biomechanical effects and can these predict a three-month clinical outcome? Arthritis Rheum. 2008;59(3):408-15.

25. Bennell KL, Bowles KA, Payne C, Cicuttini F, Williamson E, Forbes $A$, Hanna F, Davies-Tuck M, Harris A, Hinman RS. Lateral wedge insoles for medial knee osteoarthritis: 12 month randomised controlled trial. BMJ. 2011;342:d2912.

26. Laslett LL, Dore DA, Quinn SJ, Boon P, Ryan E, Winzenberg TM, Jones G. Zoledronic acid reduces knee pain and bone marrow lesions over 1 year: a randomised controlled trial. Ann Rheum Dis. 2012;71(8):1322-8.

27. Vaysbrot EE, Osani MC, Musetti MC, McAlindon TE, Bannuru RR. Are bisphosphonates efficacious in knee osteoarthritis? A meta-analysis of randomized controlled trials. Osteoarthr Cartil. 2018;26(2):154-64.

28. Cai G, Laslett LL, Aitken D, Cicuttini F, March L, Hill C, Winzenberg T, Jones G. Zoledronic acid plus methylprednisolone versus zoledronic acid or placebo in symptomatic knee osteoarthritis: a randomized controlled trial. Ther Adv Musculoskelet Dis. 2019;11. https://doi.org/10.1177/ $1759720 \times 19880054$.

29. Guymer E, Baranyay F, Wluka AE, Hanna F, Bell RJ, Davis SR, Wang Y, Cicuttini FM. A study of the prevalence and associations of subchondral bone marrow lesions in the knees of healthy, middle-aged women. Osteoarthr Cartil. 2007;15(12):1437-42.

30. Wluka AE, Hanna F, Davies-Tuck M, Wang Y, Bell RJ, Davis SR, Adams J, Cicuttini FM. Bone marrow lesions predict increase in knee cartilage defects and loss of cartilage volume in middle-aged women without knee pain over 2 years. Ann Rheum Dis. 2009;68(6):850-5.

31. Libicher M, Ivancic M, Hoffmann M, Wenz W. Early changes in experimental osteoarthritis using the Pond-Nuki dog model: technical procedure and initial results of in vivo MR imaging. Eur Radiol. 2005;15(2):390-4.

32. Lefevre N, Klouche S, Sezer HB, Gerometta A, Bohu Y, Lefevre E. The "sleeper's sign" is valid and suggestive of a medial sub-meniscal flap tear. Knee Surg Sports Traumatol Arthrosc. 2019.

33. Baker K, Grainger A, Niu J, Clancy M, Guermazi A, Crema M, Hughes L, Buckwalter J, Wooley A, Nevitt M, et al. Relation of synovitis to knee pain using contrast-enhanced MRls. Ann Rheum Dis. 2010;69(10):1779-83.

34. Wang X, Jin X, Han W, Cao Y, Halliday A, Blizzard L, Pan F, Antony B, Cicuttini $F$, Jones $G$, et al. Cross-sectional and longitudinal associations between knee joint effusion synovitis and knee pain in older adults. J Rheumatol. 2016; eeeee43(1):121-30.

35. Moisio K, Eckstein F, Chmiel JS, Guermazi A, Prasad P, Almagor O, Song J, Dunlop D, Hudelmaier M, Kothari A, et al. Denuded subchondral bone and knee pain in persons with knee osteoarthritis. Arthritis Rheum. 2009;60(12): 3703-10.

36. Zhu Z, Laslett LL, Jin X, Han W, Antony B, Wang X, Lu M, Cicuttini F, Jones G, Ding C. Association between MRI-detected osteophytes and changes in knee structures and pain in older adults: a cohort study. Osteoarthr Cartil. 2017;25(7):1084-92.

\section{Publisher's Note}

Springer Nature remains neutral with regard to jurisdictional claims in published maps and institutional affiliations.
Ready to submit your research? Choose BMC and benefit from:

- fast, convenient online submission

- thorough peer review by experienced researchers in your field

- rapid publication on acceptance

- support for research data, including large and complex data types

- gold Open Access which fosters wider collaboration and increased citations

- maximum visibility for your research: over $100 \mathrm{M}$ website views per year

At BMC, research is always in progress.

Learn more biomedcentral.com/submissions 